\title{
A relation between dynamics of coronal mass ejections and production of solar energetic particles
}

\author{
L. Kocharov ${ }^{1}$, J. Torsti ${ }^{1}$, O. C. St. Cyr ${ }^{2}$, and T. Huhtanen ${ }^{1}$ \\ 1 Space Research Laboratory, Department of Physics, University of Turku, Turku 20014, Finland \\ 2 Computational Physics Inc., NASA Goddard Space Flight Center, Greenbelt, MD 20771, USA
}

Received 29 December 2000 / Accepted 12 February 2001

\begin{abstract}
During January 1997-June 1998, the Large Angle Spectroscopic Coronagraph (LASCO) aboard the Solar and Heliospheric Observatory spacecraft (SOHO) registered 670 coronal mass ejections (CMEs). We compare two groups of the 300-800 $\mathrm{km} \mathrm{s}^{-1}$ CMEs: (i) (very) gradually accelerating CMEs with acceleration below $10 \mathrm{~m} \mathrm{~s}^{-2}$, and (ii) impulsively accelerating CMEs with acceleration $a>20 \mathrm{~m} \mathrm{~s}^{-2}$ near the Sun, continuing then with the constant speed across the LASCO field of view. An association of those CMEs with solar energetic particle (SEP) events is studied using the data of the energetic particle experiment ERNE also aboard SOHO. There were no SEP events registered in association with the first group CMEs, whereas about $8 \%$ of the second group CMEs produced an enhancement in the $\sim 10 \mathrm{MeV}$ proton flux at SOHO. This result along with a number of additional tests supports an idea that production of SEPs by the moderate speed CMEs depends not only on the final speed but also on the magnitude of acceleration that CME experiences during its liftoff. The SEP-producing CMEs are typified by impulsively accelerating CMEs accompanied by soft X-ray flares and coronal shocks.
\end{abstract}

Key words. acceleration of particles - Sun: flares - Sun: corona - Sun: particle emission

\section{Introduction}

A modern paradigm of cause and effect in solar-terrestrial physics emphasizes the role of CMEs in producing major solar energetic particle (SEP) events (Reames 1999). The correlation between SEP intensity and CME speeds was presented by Kahler et al. (1984) and updated by Reames (2000). The correlation is reported for the $2 \mathrm{MeV}$ and $20 \mathrm{MeV}$ peak proton intensities associated with CMEs in the speed range from $\sim 200$ to $\sim 2000 \mathrm{~km} \mathrm{~s}^{-1}$, with correlation coefficients $\sim 0.6-0.7$. The correlation is related to the steep enhancement in the peak intensities observed when CME speeds exceed $\sim 750 \mathrm{~km} \mathrm{~s}^{-1}$. Reames (2000) argued that the correlation coefficient $\sim 0.6-0.7$ is surprisingly high, taking into account that many factors are not considered. Kahler et al. (1999) pointed out that a considerable scattering in that correlation suggests that factors other than CME speeds play significant roles in the production of SEP events. Among others, the CME acceleration value was suggested to be a candidate factor affecting production of SEPs. Kahler et al. (1999) considered 17 poorly covered CMEs within the 650 to $850 \mathrm{~km} \mathrm{~s}^{-1}$ speed range, but the data were not sufficient to argue pro or

Send offprint requests to: L. Kocharov, e-mail: leon.kocharov@srl.utu.fi contra a distinction between accelerating versus constant speed profiles.

Case studies also can contribute to our understanding of SEP production by CMEs. Much attention was recently given to the Earthward-directed CME on January 6, 1997, which however did not produce a SEP event (e.g., Wu et al. 1997; Cane et al. 1998; Torsti et al. 1998; Fox et al. 1998; Reiner et al. 1998; Webb et al. 1998; Sheeley et al. 1999). It is interesting to compare the January 6 CME and the SEP-producing May 12, 1997 ejection. Both were Earthward-directed (halo) CMEs with low values of the plane-of-the-sky (projected) speed as observed by LASCO, 211 and $306 \mathrm{~km} \mathrm{~s}^{-1}$, respectively. The interplanetary-shock transit-speeds were significantly higher, 520 and $610 \mathrm{~km} \mathrm{~s}^{-1}$, whereas the upstream solar wind speeds respectively were 380 and $320 \mathrm{~km} \mathrm{~s}^{-1}$. The CMEs were, however, very different in the near-Sun dynamics. The January 6 CME accelerated very gradually, so that within the LASCO field of view a constantacceleration curve fits the height-time data better than a constant-velocity line does. The corresponding acceleration value is $a \sim 3 \mathrm{~m} \mathrm{~s}^{-2}$. In contrast, the May 12 ejection reached full speed close to Sun, within $\approx 4 R_{\odot}$, with very high initial acceleration $a \sim 40 \mathrm{~m} \mathrm{~s}^{-2}$, and then continued with nearly constant speed through the most of the view-field. Correspondingly, in the whole field of view the 
constant-speed fit is better than the constant-acceleration one. The May $12 \mathrm{CME}$ was accompanied by soft X-ray flare, coronal Moreton wave, and coronal type II and IV radio bursts; whereas the January 6 event was a filament eruption. There was a huge difference in corresponding SEP events: intensity of the May 12 event in the $6-12 \mathrm{MeV}$ protons exceeded by three orders of magnitude the corresponding upper limit for the January 6 event.

In present paper we perform a statistical study of relation between the acceleration that CME experiences during its liftoff and production of SEPs. We employ CME observations by the LASCO coronograph (Brueckner et al. 1995 ) and $\sim 10 \mathrm{MeV}$ proton intensities measured by the ERNE instrument (Torsti et al. 1995), both on the Solar and Heliospheric Observatory ( $\mathrm{SOHO}$ ) spacecraft. In what follows we present results of analysis of several hundreds CMEs registered during the 1.5 year period beginning in January 1997.

Being observed in the LASCO field of view, $2-30 R_{\odot}$, all CMEs fall into two categories, gradual CMEs and impulsive CMEs (Sheeley et al. 1999). Acceleration of gradual CMEs is easily observable in the LASCO field of view. This means that we know from the LASCO data an average magnitude of acceleration for the period during which CME gains the most part of the final velocity squared, $v^{2}$. In contrast, velocity of impulsive CMEs changes insignificantly across the most of the view field. The main acceleration of impulsive CMEs takes place close to Sun, the CME gains almost full $v^{2}$ below $\sim 2-4 R_{\odot}$, and continues with nearly uniform speed through $\sim 30 R_{\odot}$. For the goals of our study we must employ a value of acceleration averaged over the period during which a CME gains the bulk of $v^{2}$. For the acceleration values of gradual CMEs one can adopt the values from the LASCO CME lists ${ }^{1}$, whereas for the impulsive CMEs only a lower limit for the acceleration experienced below $\sim 2-4 R_{\odot}$ can be estimated, and such an estimate will be employed.

The typical range of the gradual CME speeds is 300 $600 \mathrm{~km} \mathrm{~s}^{-1}$ before leaving the LASCO field of view, whereas speeds of impulsive CMEs may be in excess of $750 \mathrm{~km} \mathrm{~s}^{-1}$. However, there is also a significant number of impulsive CMEs in the lower speed range, 300$700 \mathrm{~km} \mathrm{~s}^{-1}$. On the other hand, although on the whole the strongest SEP events are associated with high speed CMEs $\left(v>750 \mathrm{~km} \mathrm{~s}^{-1}\right)$, observations were reported more than once, in which significant SEP events were associated with CMEs of moderate speeds, $\sim 300-600 \mathrm{~km} \mathrm{~s}^{-1}$ (e.g., Torsti et al. 1999, 2001; Laitinen et al. 2000). That is the speed range, which both impulsive and gradual CMEs may contribute to.

Recently Torsti et al. (2001) suggested that the impulsive CME liftoff is accompanied by the coronal counterparts, soft X-ray flares and coronal shocks, and those shocks accelerate first protons escaping into the interplanetary space. Such a CME-liftoff associated acceleration

\footnotetext{
1 The lists of LASCO CMEs are available at
} http://lasco-ww. nrl.navy.mil/lasco.html probably creates a necessary condition (the seed population) for the subsequent interplanetary-shock acceleration. The coronal counterparts may be different in gradual CMEs. However, nobody has yet performed a comparative investigation of a role the two types of CMEs play in producing SEPs, at a comparable speed range but different near-Sun dynamics and probably different coronal counterparts. As a first step in such an exploration, here we address the question as to whether the SEP productivity of CMEs is an explicit function of the acceleration that CME experiences during the liftoff. This paper is aimed at testing a hypothesis that the acceleration-dependence exists along with and independently of the common speeddependence.

\section{Data analysis}

Lists showing the first appearance times and characteristics of CMEs detected in the SOHO LASCO C2/C3 images during January 1997-June 1998 contain 670 records (St. Cyr et al. 2000). In 542 cases it was possible to find polynomial fits to the CME height-time data in the 2$30 R_{\odot}$ field of view using the data reduction method described by Hundhausen et al. (1994) and St. Cyr et al. (1999). The first order (constant speed) fits are valid in 450 cases. The quadratic (constant acceleration) fits work in 92 ejections. In the latter case both the acceleration value and the final value of the CME speed are listed in the tables. We employ this set of polynomial fits to test a hypothesis that production of SEPs depends not only on the CME velocity, $v$, but also on the acceleration, $a$.

In the case of function of two arguments, $f(a, v)$, the $a$-dependence must be studied at a fixed value of $v$. Otherwise an implicit dependence via the velocity, which is in itself dependent on acceleration, $f(v(a))$, might be mistaken for the explicit dependence $f(a, v=$ constant). This reasoning is equally valid for a statistical study, with the correction that values $v$ and $a$ must be replaced with a set of bins in those variables. Width of the bins is dictated by the statistics available. We adopt $3 \times 3$ bins in the velocity-acceleration plane. There are three bins in $v:(V 1) v<300 \mathrm{~km} \mathrm{~s}^{-1}$; $(V 2) 300<v<800 \mathrm{kms}^{-1}$; and $(V 3) v>800 \mathrm{~km} \mathrm{~s}^{-1}$. For the three bins in acceleration we employ: $(A 1)$ a subclass of accelerating LASCO CMEs with $a<10 \mathrm{~m} \mathrm{~s}^{-2}$ (note that in all registered cases $\left.a>2 \mathrm{~m} \mathrm{~s}^{-2}\right)$; (A2) a subclass of accelerating LASCO CMEs with $a \geq 10 \mathrm{~m} \mathrm{~s}^{-2}$ (note that in the middle velocitybin $85 \%$ of them have $a<20 \mathrm{~m} \mathrm{~s}^{-2}$ ); and (A3) constantspeed LASCO CMEs, which experience a strong acceleration below $\sim 2-4 R_{\odot}$. The estimated lower limit for the acceleration of the latter group CMEs is $20 \mathrm{~m} \mathrm{~s}^{-2}$. A poor statistics of accelerating CMEs in the high velocity range, $V 3$, and the low expectation of SEP production by the low velocity CMEs in the $V 1$ range send us in a search for the $a$-dependence in the $V 2$ range only ${ }^{2}$.

\footnotetext{
${ }^{2}$ Restricting the CME speed range for the goals of a statistical study was previously employed by Kahler et al. (1999).
} 
Table 1. List of impulsive CMEs associated with SEP events

\begin{tabular}{|c|c|c|c|c|c|c|c|}
\hline \multirow[t]{2}{*}{ Date } & \multicolumn{2}{|c|}{$\mathrm{CME}$} & \multicolumn{4}{|c|}{ Flare } & \multirow[t]{2}{*}{ Remarks } \\
\hline & Time $^{a}$ & Speed & $\mathrm{X}-\mathrm{ray}^{\mathrm{b}}$ & $\mathrm{H} \alpha^{\mathrm{c}}$ & Type $\mathrm{II}^{\mathrm{c}}$ & Type IV & \\
\hline$\overline{1997 / 04 / 01}$ & $15: 18$ & 296 & $14: 51$ & $14: 52$ & $13: 49$ & $13: 46$ & \\
\hline $1997 / 05 / 12$ & $06: 30$ & 306 & $04: 55$ & 04:44 & $04: 58$ & $05: 10$ & \\
\hline $1997 / 05 / 21$ & $21: 00$ & 303 & $20: 15$ & 20:08 & $20: 10$ & $20: 27$ & \\
\hline $1997 / 07 / 25$ & 21:01 & 632 & $19: 41$ & $20: 11$ & $20: 24$ & $20: 34$ & \\
\hline $1997 / 09 / 20$ & $10: 20$ & 789 & $10: 44$ & & & & \\
\hline $1997 / 10 / 12$ & $05: 35$ & 728 & $06: 54$ & & $06: 13$ & & Or CME at 06:26 UT, $823 \mathrm{~km} \mathrm{~s}^{-1}$. \\
\hline $1997 / 10 / 16$ & $22: 29$ & 594 & $21: 47$ & & & & \\
\hline $1997 / 10 / 21$ & $18: 03$ & 465 & $17: 54$ & $17: 33$ & & & \\
\hline $1997 / 11 / 03$ & $05: 28$ & 297 & $04: 38$ & $04: 34$ & $04: 38$ & $04: 56$ & Probably two close CME+SEP events. \\
\hline $1997 / 11 / 04$ & $04: 20$ & 645 & $04: 16$ & 03:03 & 06:08 & $06: 11$ & Or CME at 06:10 UT, $830 \mathrm{~km} \mathrm{~s}^{-1}$ \\
\hline $1997 / 11 / 13$ & $22: 25$ & 449 & $23: 40$ & & & & \\
\hline $1997 / 11 / 17$ & $08: 27$ & 767 & 08:03 & & & & Probably two close CME+SEP events. \\
\hline 1998/01/02 & $23: 28$ & 446 & $23: 35^{\mathrm{c}}$ & & & & \\
\hline $1998 / 01 / 19$ & $07: 33$ & 358 & $07: 11$ & & $06: 59$ & & Probably two close CME+SEP events. \\
\hline $1998 / 01 / 26$ & $23: 27$ & 446 & $22: 35$ & $22: 24$ & $22: 31$ & & \\
\hline $1998 / 03 / 25$ & $13: 14$ & 762 & $11: 49$ & $11: 46$ & & & \\
\hline $1998 / 05 / 14$ & $03: 55$ & 373 & 03:01 & & $03: 24$ & & \\
\hline 1998/05/19 & $10: 27$ & 796 & $10: 15$ & & 09:51 & & \\
\hline $1998 / 05 / 30$ & $23: 28$ & 488 & & & $22: 48$ & & \\
\hline
\end{tabular}

In the case of several solar events of the same type associated with a CME, only the nearest event is shown.

Speed of CME is in $\mathrm{km} \mathrm{s}^{-1}$.

a First appearance time, UT.

b Maximum time, UT.

c Start time, UT.

In an effort to observe even not very strong effect of acceleration we initially select CMEs most separated in $a$, groups $A 1$ and $A 3$. Then the $A 2$ group will be also employed. Thus we start with comparison of the SEP-event occurrences in association with two groups of CMEs: 1) $V 2 \_A 3$, constant speed CMEs with (two digit rounded) speed values in the range from $300 \mathrm{~km} \mathrm{~s}^{-1}$ through $800 \mathrm{kms}^{-1}$; and 2) $V 2 \_A 1$, gradually accelerating CMEs in the same speed range with the acceleration value $a<10 \mathrm{~m} \mathrm{~s}^{-2}$. We have $253 \mathrm{CMEs}$ of the first type and $43 \mathrm{CMEs}$ of the second type. Hereinafter the first type CMEs are termed impulsive, and the second type CMEs are termed gradual for short, despite this definition of the classes does not exactly coincide with the definition by Sheeley et al. (1999). Figures 1 and 2 show distributions of the impulsive and gradual CMEs over the speed and over the angular width. The SEP-associated impulsive CMEs and all gradual CMEs are listed in Tables 1 and 2 , respectively.

The CME associated SEP-events were selected using the ERNE 1-100 MeV proton data. In the first stage of selection we find all significant enhancements in the 6.412.7 MeV proton channel. Then the neighboring energy channels are used (i) for revealing simultaneous increases in different energy channels, which obviously are of a local origin and should be dropped, and (ii) for refining solar injection time of the first protons observed above the background $^{3}$. In the last stage an association of the SEP event with a CME is established, if the first proton injection time does not differ by more than $3 \mathrm{~h}$ from the first appearance time of a CME. The $3 \mathrm{~h}$ criterion is adopted because the rising phase of the SEP event may extend until the time of the CME arrival at $\approx 10 R_{\odot}$ (Kahler 1994), and the pre-event background may be enhanced. The list of CMEs associated with proton events is given in Table 1. It turns out that all 19 SEP-associated CMEs are impulsive. None of gradual CMEs (Table 2) was accompanied by $\sim 10 \mathrm{MeV}$ proton event in ERNE.

We also studied association of different groups of CMEs with (i) soft X-ray flares, (ii) $\mathrm{H} \alpha$ flares, (iii) metric type II radio bursts, and (iv) type IV bursts, using lists available in Solar-Geophysical Data $(1997,1998)$. The association of a solar event (i-iv) with the CME is established if in the $1.5 \mathrm{~h}$ vicinity of the first appearance time of CME we find at least one of the following times of the solar event: start or maximum time of soft X-ray flare, start time of $\mathrm{H} \alpha$ flare, start time of type II/IV radio burst.

\footnotetext{
${ }^{3}$ Note that some particle events still may be missed, because of high background level from previous events, or in the case of occurrence on the backside of the Sun or at far-eastern longitudes. However, we see no reasons to suspect that the obscuring factors affect intervals of gradual CMEs to a greater extent than intervals of impulsive CMEs, whereas our method is based on the comparison of the two classes.
} 
Table 2. List of gradual CMEs $\left(a<10 \mathrm{~m} \mathrm{~s}^{-2}\right)$

\begin{tabular}{|c|c|c|c|c|c|}
\hline \multirow[t]{2}{*}{ Date } & \multicolumn{3}{|c|}{$\mathrm{CME}$} & \multicolumn{2}{|c|}{ Flare } \\
\hline & Time $^{\mathrm{a}}$ & Speed & Accel. & $\mathrm{X}-$ ray $^{\mathrm{b}}$ & $\mathrm{H} \alpha^{\mathrm{c}}$ \\
\hline $1997 / 01 / 18$ & $02: 30$ & 357 & 5.7 & & \\
\hline $1997 / 02 / 03$ & $18: 18$ & 524 & 8.4 & $18: 38$ & \\
\hline $1997 / 02 / 11$ & & 296 & 5.7 & & \\
\hline 1997/03/09 & 04:30 & 604 & 6.8 & $03: 40^{\mathrm{c}}$ & \\
\hline $1997 / 03 / 28$ & $02: 55$ & 398 & 3.2 & $02: 25$ & \\
\hline $1997 / 04 / 04$ & 05:09 & 326 & 4.6 & & \\
\hline $1997 / 05 / 01$ & $19: 22$ & 360 & 3.4 & & \\
\hline $1997 / 05 / 04$ & $22: 57$ & 575 & 7.5 & & \\
\hline 1997/06/01 & $07: 30$ & 360 & 8.0 & & \\
\hline $1997 / 06 / 16$ & $20: 31$ & 475 & 6.2 & & \\
\hline $1997 / 07 / 14$ & $08: 51$ & 320 & 2.8 & & \\
\hline 1997/08/12 & $11: 33$ & 330 & 4.3 & & $12: 40$ \\
\hline 1997/09/17 & $20: 28$ & 487 & 6.9 & & \\
\hline 1997/09/19 & 01:29 & 460 & 5.2 & $01: 50$ & 01:47 \\
\hline $1997 / 09 / 27$ & $12: 35$ & 363 & 4.2 & & \\
\hline $1997 / 10 / 03$ & $19: 35$ & 325 & 3.0 & $18: 23$ & \\
\hline $1997 / 10 / 06$ & $15: 28$ & 523 & 9.2 & & \\
\hline $1997 / 10 / 07$ & $17: 28$ & 324 & 2.2 & $18: 25$ & \\
\hline $1997 / 11 / 01$ & $20: 11$ & 411 & 6.0 & & \\
\hline $1997 / 11 / 08$ & 08:59 & 303 & 3.2 & 08:19 & \\
\hline $1997 / 11 / 11$ & $17: 37$ & 412 & 5.8 & $17: 21$ & \\
\hline $1997 / 12 / 05$ & $08: 27$ & 543 & 4.0 & & \\
\hline $1997 / 12 / 12$ & $01: 27$ & 345 & 6.6 & $00: 54$ & \\
\hline $1997 / 12 / 26$ & 02:31 & 347 & 4.7 & & \\
\hline $1998 / 01 / 28$ & $14: 56$ & 408 & 3.8 & & \\
\hline $1998 / 01 / 31$ & $12: 41$ & 521 & 7.7 & & \\
\hline 1998/02/04 & 17:02 & 494 & 5.2 & $17: 51$ & \\
\hline 1998/03/01 & $10: 03$ & 316 & 2.4 & 09:33 & \\
\hline 1998/03/01 & 10:03 & 514 & 6.0 & 11:09 & 11:07 \\
\hline $1998 / 03 / 23$ & 09:33 & 588 & 8.0 & & $08: 32$ \\
\hline 1998/03/31 & $21: 23$ & 647 & 9.6 & & \\
\hline 1998/04/08 & $03: 27$ & 564 & 9.5 & 04:06 & \\
\hline $1998 / 05 / 06$ & $02: 28$ & 511 & 6.0 & & $02: 25$ \\
\hline $1998 / 05 / 07$ & 01:30 & 390 & 5.6 & 01:10 & 01:09 \\
\hline $1998 / 05 / 29$ & $22: 30$ & 755 & 9.6 & $21: 32$ & $23: 42$ \\
\hline $1998 / 05 / 30$ & $02: 27$ & 501 & 7.6 & & \\
\hline $1998 / 06 / 05$ & $12: 01$ & 412 & 7.8 & & \\
\hline 1998/06/09 & $09: 27$ & 314 & 7.9 & 08:39 & \\
\hline 1998/06/09 & $11: 27$ & 508 & 6.6 & & \\
\hline 1998/06/13 & $02: 27$ & 670 & 9.8 & $01: 55$ & 03:09 \\
\hline $1998 / 06 / 15$ & $14: 55$ & 493 & 5.6 & & $13: 25$ \\
\hline 1998/06/16 & $05: 27$ & 554 & 8.1 & & \\
\hline $1998 / 06 / 22$ & $07: 34$ & 417 & 7.0 & $08: 37$ & \\
\hline
\end{tabular}

In the case of several solar events of the same type associated with a CME, only the nearest event is shown. Speed and acceleration of CME are in $\mathrm{km} \mathrm{s}^{-1}$ and $\mathrm{m} \mathrm{s}^{-2}$, respectively.

a First appearance time, UT.

b Maximum time, UT.

c Start time, UT.

The CME-associated solar events are also listed in Tables 1 and 2. Different associations are summarized in Fig. 3 for all gradual CMEs, for all impulsive CMEs, and

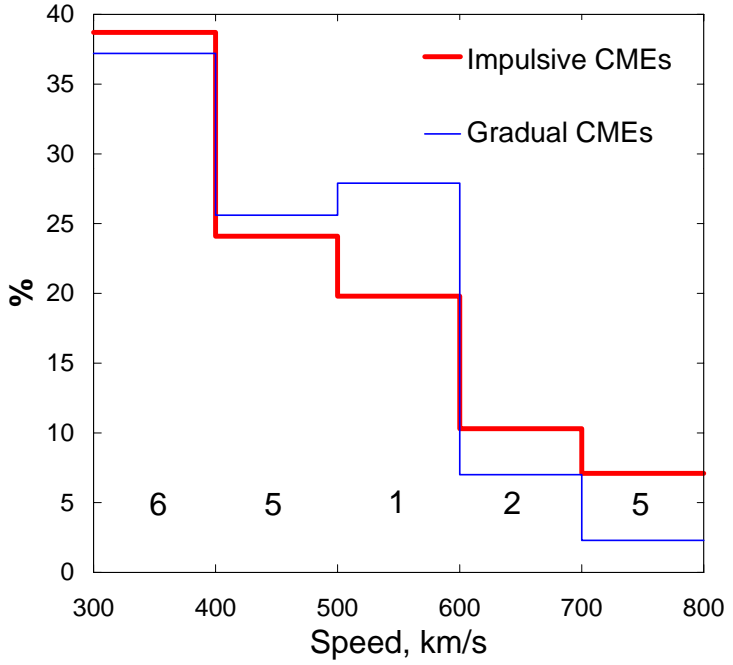

Fig. 1. Speed distributions of impulsive and gradual $(a<$ $10 \mathrm{~m} \mathrm{~s}^{-2}$ ) CMEs. Total statistics are 253 and 43 CMEs, respectively. The row " $6,5,1,2,5$ " shows numbers of SEP events associated with impulsive CMEs in the corresponding sub-bins

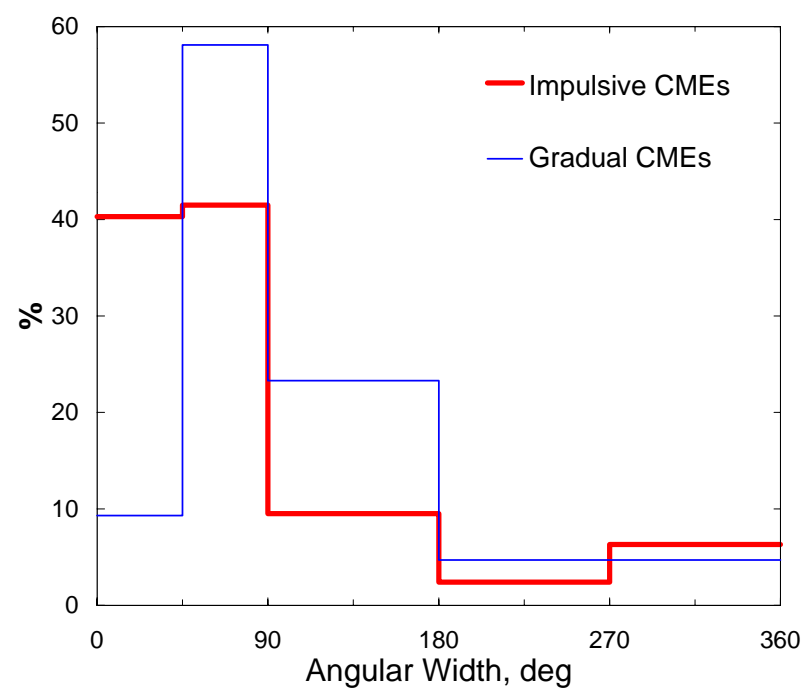

Fig. 2. Width distributions of impulsive and gradual $(a<$ $10 \mathrm{~m} \mathrm{~s}^{-2}$ ) CMEs. Total statistics are 253 and $43 \mathrm{CMEs}$, respectively

also for the impulsive CMEs associated with proton events (SEP-producing CMEs).

\section{Discussion}

Our search revealed $n_{\mathrm{i}}=19$ SEP-associated CMEs (Table 1) among the total $N_{\mathrm{i}}=253$ impulsive CMEs, whereas no SEP-associated CMEs $\left(n_{\mathrm{g}}=0\right)$ were found among $N_{\mathrm{g}}=43$ gradual CMEs (Table 2). To check whether this is by a random chance or not, one can employ a statistics

$\chi^{2}=\left(n_{\mathrm{i}} N_{\mathrm{g}}-n_{\mathrm{g}} N_{\mathrm{i}}\right)^{2}\left[N_{\mathrm{i}} N_{\mathrm{g}}\left(n_{\mathrm{i}}+n_{\mathrm{g}}\right)\right]^{-1}$

at one degree of freedom (Bolshev \& Smirnov 1983). This criterion verifies a conclusion that probabilities to observe SEP events in association with impulsive CMEs 


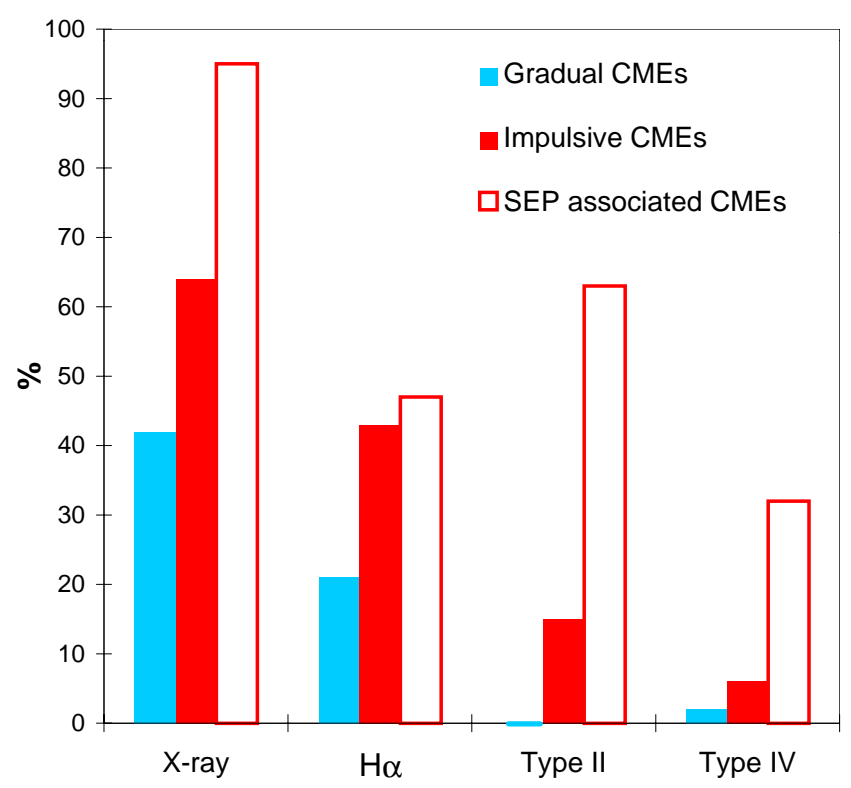

Fig. 3. Percentage of CMEs associated with soft X-ray and $\mathrm{H} \alpha$ flares, type II and IV radio bursts for the different CME groups. Total statistics are 43, 100, and 19 for the gradual $(a<$ $10 \mathrm{~m} \mathrm{~s}^{-2}$ ) CMEs, impulsive CMEs, and SEP-associated CMEs, respectively. The 100 impulsive CMEs have been selected by a random number generator from the total 253 impulsive CMEs

and in association with gradual $\left(<10 \mathrm{~m} \mathrm{~s}^{-2}\right)$ CMEs are significantly different. The straightforward calculation of $\chi^{2}$ yields at this stage the Estimated Confidence Level $\mathrm{ECL}=93 \%^{4}$.

We have compared gradual CMEs with acceleration $a<10 \mathrm{~m} \mathrm{~s}^{-2}$ and impulsive CMEs, both in the speed range $300 \leq v \leq 800 \mathrm{~km} \mathrm{~s}^{-1}$. The comparison reveals a significant difference in association of the two groups of CMEs with $\sim 10 \mathrm{MeV}$ proton events. The gradually accelerating CMEs did not produce SEP events, while about $8 \%$ of impulsive CMEs were associated with SEP events at SOHO. Note that the latter percentage accounts only for the SEP events observable at a given solar longitude. The longitude-integrated probability is significantly higher, most likely above $70 \%$, because the average size of the impulsive CMEs is only $77^{\circ}$. Note that for gradual CMEs with $a<10 \mathrm{~m} \mathrm{~s}^{-2}\left(15 \mathrm{~m} \mathrm{~s}^{-2}\right)$ the mean size is $93^{\circ}\left(97^{\circ}\right)$. The difference is revealed between frequencies of the SEP-event occurrences in the $V 2 \_A 1$ and $V 2 \_A 3$ CME bins, at the confidence level ECL $=93 \%$. In additional set of calculations, we tested sensitivity of the result to the biases in the speed/size distributions, and to the choice of the bin boundaries. Effect of the $V 2 \_A 2$ data-bin employment was also investigated.

\footnotetext{
4 The estimate may be also done in less rigorous but more transparent manner. At probability $P=19 / 253$ estimated from the statistics of impulsive CMEs, the probability of the event that no SEP enhancement has occurred in 43 trials is $(1-P)^{43}=3.5 \%$. Hence, a probability to observe a SEP event in association with gradual CME is significantly less than that for impulsive CMEs, at ECL $\approx 96.5 \%$.
}

Previously reported correlation between SEP intensities and CME speeds finds its counterpart in a speed dependence of probability to observe SEP event in association with a given CME. This is evident from comparison of the event statistics in different velocity bins, $V 1 \_A 3$ vs. $V 2 \_A 3$ vs. $V 3 \_A 3$. In the medium velocity bin $V 2 \_A 3$ we find 19 events per $253 \mathrm{CMEs}$, whereas there are 14 SEP events per 38 CMEs in the high speed bin $V 3 \_A 3$, and only one event per 159 CMEs in the lowest speed bin $V 1 \_A 3$. Thus the percentage of SEP events per one CME rises from less or about $1 \%$ for the low speed CMEs, $<300 \mathrm{~km} \mathrm{~s}^{-1}$, to almost $40 \%$ for high speed CMEs, $>800 \mathrm{~km} \mathrm{~s}^{-1}$. Such a tendency, if it holds inside the $V 2$ range, may slightly affect our comparison of different acceleration bins, $V 2 \_A 1$ vs. $V 2 \_A 3$, if velocity distributions inside the bins are biased in respect to one another.

One can note in Fig. 1 approximately three fold excess in the number of impulsive CMEs in the highest speed subbin, $700-800 \mathrm{~km} \mathrm{~s}^{-1}$, as compared to the gradual CMEs. In order to examine whether this bias can affect our result, we dropped three SEP events of five corresponding to this bin, along with the corresponding portion of impulsive $700-800 \mathrm{~km} \mathrm{~s}^{-1}$ CMEs. Such a manipulation alone shifts the estimated confidence level for the difference between the $V 2 \_A 1$ and $V 2 \_A 3$ CMEs from $93 \%$ down to $90 \%$, but it should be also taken into account that on the whole gradual CMEs are wider than the impulsive ejections (Fig. 2). Correspondingly SEPs from gradual CMEs might be more easily detected at a spacecraft. A straightforward correction for the difference in the CME solid angles shifts the ECL value from $90 \%$ up to $95 \%$. Hence, accounting for the biases in the speed/size distributions finally rises the confidence level to ECL $=95 \%$.

We have additionally analyzed a possible role of the middle acceleration-bin, $V 2 \_A 2$, which contains accelerating LASCO CMEs with acceleration in excess of $10 \mathrm{~m} \mathrm{~s}^{-2}$. There are 20 CMEs and 3 SEP events in $V 2 \_A 2$, whereas in the $V 2 \_A 3$ bin we have 253 CMEs and 19 SEP events, and 43 CMEs and no SEP events in $V 2 \_A 1$. Appearance of SEP events in $V 2 \_A 2$ may indicate increase in the SEPevent probability as the CME acceleration increases inside the class of accelerating LASCO CMEs. Comparable frequencies of SEP-events in $V 2 \_A 2$ and $V 2 \_A 3$ bins justify integration of the bins. If one had merged all $a>10 \mathrm{~m} \mathrm{~s}^{-2}$ CMEs into the enlarged bin, $V 2 \_A 2+V 2 \_A 3$, and compared $a<10$ and $a>10 \mathrm{~m} \mathrm{~s}^{-2}$ CMEs, the estimated confidence level would be close to the ECL value for comparison $V 2 \_A 1$ vs. $V 2 \_A 3$. We also employed the bin boundary $a=15 \mathrm{~m} \mathrm{~s}^{-2}$, instead of our basic $a=10 \mathrm{~m} \mathrm{~s}^{-2}$, and compared gradual CMEs with $a<15 \mathrm{~ms}^{-2}$ and all $a>15 \mathrm{~m} \mathrm{~s}^{-2}$ CMEs. In this case, 12 more CMEs fall into the slow acceleration group, including a single SEP-event, so that we have 1 SEP-event per 55 slow-acceleration CMEs and 21 SEP-events per 261 fast-acceleration CMEs. At this division, a bias in the speed distributions is absent, but the angular size difference persists, and the resultant $\mathrm{ECL}=97 \%$. 
On the other hand, the merge of bins $V 2 \_A 2$ and $V 2 \_A 1$ a priori removes the acceleration dependence in a very wide region $a \sim 2-20 \mathrm{~m} \mathrm{~s}^{-2}$ and should obscure the general trend observable in the three bin succession. Despite this argument against the merge, one may attempt to compare the enlarged bin $V 2_{-} A 1+V 2_{-} A 2$ and $V 2_{-} A 3$, arguing that $V 2 \_A 1+V 2 \_A 2$ represents a separate class of LASCO CMEs according to the visual "constant-speed vs. accelerating" CME classification. Such a comparison, at velocity/width biases corrected ${ }^{5}$, yields a conclusion that the frequency of SEP-events associated with accelerating CMEs and the frequency of SEP-events associated with constant-speed CMEs are different at $\mathrm{ECL}=91 \%$. A number of tests performed indicate that on the whole our basic result is qualitatively stable and suggests increase in the frequency of SEP events as the CME acceleration increases above $\sim 10 \mathrm{~m} \mathrm{~s}^{-2}$.

The $\chi^{2}$-test revealed significant distinctions also between the CME $\rightarrow$ flare associations (Fig. 3). Degrees of association in different pairs, gradual/impulsive/SEPassociated CMEs versus X-ray/type II bursts, are significantly different at ECL $=85.0-100.0 \%$. We find that the moderate-speed impulsive CMEs associated with SEPs are also highly associated with X-ray flares and type II radio bursts. Thus, the typical SEP-producing CME is that impulsively accelerated at heliocentric distances $<2-4 R_{\odot}$ in association with soft X-ray flare and coronal shocks. We would like to stress that we do not examine association of flares and SEPs. Our statistics is related to the association of different groups of CMEs with X-ray flares and type II/IV radio burst. Among others, we have considered a group of CMEs which were accompanied by SEP events at SOHO. It turns out that these SEP-producing CMEs are highly associated with X-ray flares, despite only a small portion of flares is associated with SEP events.

In early 1980s, MacQueen \& Fisher (1983) studied 12 CMEs and found that flare-related CMEs moved at constant speeds, whereas prominence-related mass ejections accelerated. A more comprehensive study that determines accelerations for CMEs based on SMM and Mauna Loa observations confirms that conclusion (St. Cyr et al. 1999). Recent investigations with LASCO (Sheeley et al. 1999) suggest two principal types of CMEs: gradually accelerating CMEs and impulsive CMEs. Impulsive CMEs, often associated with flares and Moreton waves, move uniformly across the LASCO field of view. Hence, CMEs of this kind should experience a strong acceleration when getting a full speed during a short period below $\sim 2-4 R_{\odot}$.

Kahler et al. (1999) proposed the CME acceleration value as a candidate factor affecting production of

\footnotetext{
${ }^{5}$ Correction for the velocity distribution bias means that the number of impulsive CMEs in each sub-bin of Fig. 1 is multiplied by a correction factor to make velocity distributions of impulsive and gradual CMEs exactly identical. The number of associated SEP events in each sub-bin is changed by the same factor. Correction for the angular width bias means that the total number of SEP events is multiplied by the gradualto-impulsive CME solid-angle ratio.
}

$>20 \mathrm{MeV}$ protons, because "an accelerating CME may produce a shock later than a CME with a constant speed". Based on this kinematics-type explanation and on the result of the present statistical study, a question should be raised as to why it is so crucial for shock to appear close to Sun. In this respect two reasons might be considered: (i) a constant-speed shock maintains high Mach numbers predominantly close to Sun, and (ii) a seed particle/wave population for the (moderate-speed) CME-shock acceleration is available only near the Sun. We see no arguments in favour of the first item over the second one, because Alfvén speed does not decrease towards the Sun. On the other hand, Torsti et al. (2001) analyzed the May 9, 1999 SEP event associated with impulsive CME $\left(540 \mathrm{~km} \mathrm{~s}^{-1}\right)$, X-ray flare, and continuum radio burst in metric band. The case study supports a kind of "closed-chain scenario": an early rise of CME triggers the X-ray flare that starts a coronal wave, which in its turn initiates proton acceleration apart from the flare site. This first proton acceleration is triggered by the coronal counterparts of CME but followed by the long-term acceleration at the CME-driven shock in the interplanetary medium. It was suggested that the first acceleration may provide a seed population for the second one. Note that in the case of the May 9, 1999 event, neither of the accelerations was identified with the flaresite acceleration. However, presently available case studies are still not sufficient to decide whether such a scenario is typical for the impulsive CMEs of a moderate speed.

We have studied SEP production by the 300$800 \mathrm{~km} \mathrm{~s}^{-1} \mathrm{CMEs}$, and conclude that data in hand support the following

1. The near-Sun dynamics of CME is a significant factor contributing to the intensities of $\sim 10 \mathrm{MeV}$ proton events;

2. A typical SEP-producing CME experiences fast acceleration close to Sun in association with soft X-ray flare and coronal shocks;

3 . The CMEs (very) gradually accelerating across the $2-$ $30 R_{\odot}$ field of view with acceleration $2-10 \mathrm{~m} \mathrm{~s}^{-2}$ are less likely to produce $>10 \mathrm{MeV}$ SEP events.

The conclusions have been drawn with a statistical method at the estimated confidence level of about $97 \%$. These results indicate that at least in the moderate speed range the CME acceleration must be further considered as a significant factor affecting production of solar energetic particles along with the CME speed. The joint analysis of the LASCO and ERNE data-sets brings out one more promising direction for the SEP-CME investigations.

Acknowledgements. The ERNE team thanks Academy of Finland for the grant. The SOHO is an international cooperation project between ESA and NASA.

\section{References}

Bolshev, L. N., \& Smirnov, N. V. 1983, Tables of mathematical statistics, Nauka, Moscow, 359 
Brueckner, G. E., Howard, R. A., Koomen, M. J., et al. 1995, Solar-Geophysical Data $1997 \quad \& \quad 1998, \quad$ NOAA, Space Sol. Phys., 162, 357

Environment Center, Boulder, Colorado

Cane, H. V., Richardson, I. G., \& St. Cyr, O. C. 1998, GRL, Sheeley Jr., N. R., Walters, J. H., Wang, Y.-M., \& Howard, 25, 2517; Correction: 26, 2149 R. A. 1999, JGR, 104, 24739

Fox, N. J., Peredo, M., \& Thompson, B. J. 1998, GRL, 25, St. Cyr, O. C., Burkepile, J. T., Hundhausen, A. J., \& Lecinski, 2461

Hundhausen, A. J., Burkepile, J. T., \& St. Cyr, O. C. 1994, JGR, 99, 6543

A. R. 1999, JGR, 104, 12493

St. Cyr, O. C., Howard, R. A., Sheeley Jr., N. R., et al. 2000, JGR, 105, 18169

Kahler, S. W., Sheeley Jr., N. R., Howard, R. A., et al. 1984, Torsti, J., Valtonen, E., Lumme, M., et al. 1995, Sol. Phys., JGR, 89, 9683

Kahler, S. 1994, ApJ, 428, 837

Kahler, S., Burkepile, J. T., \& Reames, D. V. 1999, Proc. 26th Internat. Cosmic Ray Conf., Salt Lake City, 6, 248

Laitinen, T., Klein, K.-L., Kocharov, L., et al. 2000, A\&A, 360, 729

MacQueen, R. M., \& Fisher, R. R. 1983, Sol. Phys., 89, 89

Reames, D. V. 1999, Space Sci. Rev., 90, 413 162,505

Torsti, J., Anttila, A., Kocharov, L., et al. 1998, GRL, 25, 2525

Torsti, J., Kocharov, L., Teittinen, M., et al. 1999, JGR, 104, NO. A5, 9903

Torsti, J., Kocharov, L., Innes, D. E., et al. 2001, A\&A, 365, 198

Webb, D. F., Cliver, E. W., Gopalswamy, N., Hudson, H. S., \& St. Cyr, O. C. 1998, GRL, 25, 2469

Reames, D. V. 2000, in 26th Int. Cosmic Ray Conf., ed. B. L. Dingus, D. B. Kieda, \& M. H. Salamon, New York: AIP Conf. Proc. 516, 289

Wu, S. T., Guo, W. P., Michels, D. J., et al. 1997, in Proc. 5th SOHO Workshop, ed. A. Wilson, Noordwijk: ESA SP-404, 739

Reiner, M. J., Kaiser, M. L., Fainberg, J., et al. 1998, GRL, 25,2493 